\title{
VR BBS Using Immersive Virtual Environment
}

\author{
Satoshi Oonuki \\ University of Tsukuba \\ oonuki@gil.cs.tsukuba.ac.jp
}

\author{
Tetsuro Ogi \\ University of Tsukuba \\ tetsu@cc.tsukuba.ac.jp
}

\begin{abstract}
In this research, VR BBS was developed. In this system, a user can post a photograph with a comment from cellular phone. And the users can see and reply to the photograph and comment by using common BBS on the cellular phone and PC. In addition, the user can also use BBS function in the virtual world. In this study, an immersive projection display was used to experience the virtual world. The virtual world is constructed by placing the photograph images at the position measured by GPS function of the cellular phone. In the virtual world, the user can see photographs and comments on the maps, and reply to them while walking through the world. This system enables users to share information mutually using the cellular phone, PC, and immersive projection display. This system also has a feature that the virtual world would be automatically extended when many users use this system.
\end{abstract}

\section{Introduction}

In recent years, a lot of web-based services have been provided according to the spread of the Internet. Although in early services, the user had only received information which was provided by the web sites, in the current systems, the user can send and receive information using the BBS (Bulletin Board System) or Weblog functions. However in the ordinary BBS, it is difficult for the users to communicate with each other while sharing the experience, because they can post the comment using only the text information.

On the other hand, data sharing sites such as the Youtube [1] and Flickr [2] have become popular. In these web sites, when the user uploads his video or photograph, other users can see it and write comments on the web page. These video and photo sharing sites are acceptable for the user compared to the ordinary BBS in which only the text information is used. Then, the users can communicate with each other while sharing the photo and video data. However, in these systems, it is difficult to manage the uploaded contents efficiently.

Recently, various services using the map information, such as the Google Maps [3] have been constructed, because API of the Google Maps is open to the public. In the photo sharing site using the Google Maps, users can visually recognize the positional relationship among photographs which are placed on the map. These sites in which user can post photographs and messages on the map are called map BBS. KASHIMIR [4] is the example of the map BBS.

However, in the web applications used on the PC, it is difficult for the users to recognize the detailed information when a large amount of photographs are displayed on the map. Therefore, in most photo sharing sites, the number of displayed photographs is limited according to the area of the displayed map. For example, when the map is zoomed in, the photographs that were not displayed till then appear though the display area is decreased. Then, it would become difficult for the user to understand the positional relationship among photograph data.

The purpose of this research is constructing the VR BBS, in which the user can post a comment or a message while experiencing the virtual world. In addition, this system aims at realizing the immersive information space where the users can share the information with the person in the outdoor field.

\section{Map BBS Function}

First, in this study, we have developed map BBS function running on the $\mathrm{PC}$ using the Google Maps API. In the map BBS function, each user can upload the photographs and manage them by classifying them into categories. In order to use the map BBS function, the user's account must be registered, and when the user logs in, personal page is displayed. Then, the user put a marker on the map by clicking the corresponding position, and uploads the photograph by filling in the items of file name of the photograph, title and comment on the form. The user can change the location of the registered photograph on the map or he 
can change the comment on the editing page. By using the category, the user can classify the photographs into some groups such as travel or restaurant, and he can use BBS in the sense of writing a diary. In addition, the user who is not registered can also post a comment to other user's photograph.

The operation flow of the map BBS function is shown in from Figure 1 to Figure 5. First, when a user logs in the system, an initial personal page is displayed on the web. When the user clicks the photograph upload button, registration form of the photograph is displayed. And the user can put a marker on the map by clicking the corresponding position on it. After that, the user registers photographs by filling in the items of file name, title, and comment on the registration form (Figure 1). In this case, the user can register several photographs at the same position on the map.

Figure 2 shows the screenshot when the photograph was registered. Markers are placed on the map, and a photograph is displayed in a small window when the user clicks a marker. When two or more photographs are registered at the same position on the map, a largish marker is displayed. And when the largish marker is clicked by the user, all the photographs registered at the position are displayed.

When the user wants to change the registered data, he can edit it on the editing page as shown in Figure 3. For example, when he wants to change the category name, he can edit it on the editing page. If the category name were changed, all the photographs that are classified into it would change the property of the category name. These categories are classified into two kinds of groups, one is open to public and the other is private. When the user specified the photographs as public, other user would be able to access and browse them freely.

When the user finds an interesting photograph, he can make a bookmark on it. These photographs are managed in the bookmark list and the order of the selected photographs in the list can be changed on the bookmark editing page (Figure 4). This bookmark list is also classified into public or private in order to specify the accessibility of other users. The registered photographs are displayed on the map by specifying the category or the registration date. The photograph that is placed in the virtual world is also specified by using the category. Details are described in the next chapter.

On the comment page, the user can write a comment for the other users' photographs and upload it using the comment upload form (Figure 5).

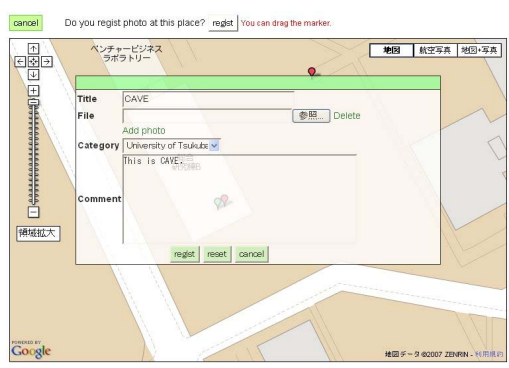

Figure 1. Registration form of photograph

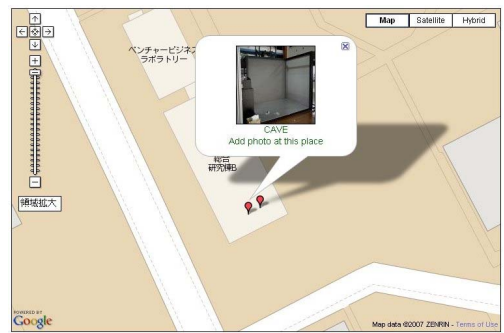

Figure 2. Photograph registered on the map

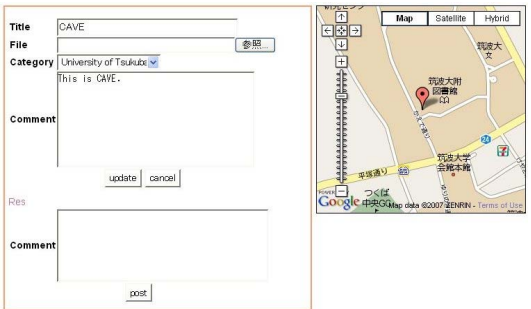

Figure 3. Edit of photograph information

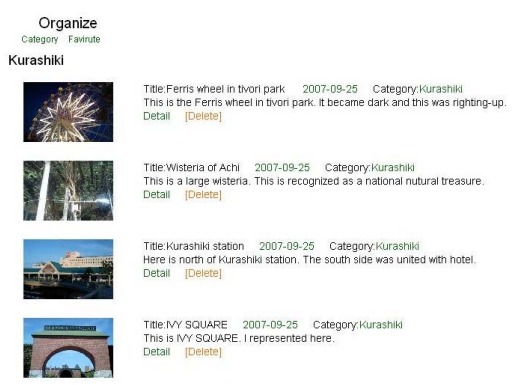

Figure 4. Edit of bookmark

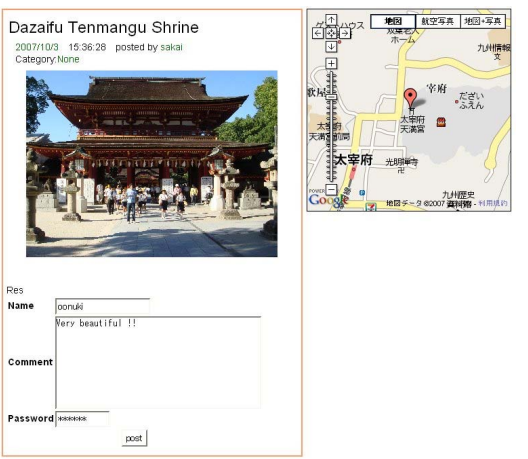

Figure 5. Comment upload form 


\section{Cellular Phone}

Though the user can make a record of the travel by uploading the photographs and comments using the map BBS function, he cannot always carry a PC. Therefore, it is difficult for the traveler to post a photograph from the place where he is visiting. In this system, a function of sending a photograph and a comment using a cellular phone was introduced. Recent cellular phone includes various kinds of functions such as Internet access, digital camera and GPS. In this study, CASIO G'zOne cellular phone for AU was used. When the user in the outdoors takes a photograph using the digital camera function of the cellular phone, the current position is measured by the GPS function and it is attached to the photograph as Exif data. Then, he sends the photograph and position data to the server using the e-mail function. And when the e-mail reaches the server computer, it is analyzed by the parser program to take out the photograph and the position data, and then they are stored in the database. In this method, since the photograph images sent from the cellular phone are stored in the same database as the photographs that are registered from the PC, they can be displayed on the map automatically.

However, since the Google Maps applications cannot be used on the cellular phone, the access page for the cellular phone where the user can browse the photographs and comments without using the Google Maps was made. On this web page, the title, photograph and comment are displayed. And the user can also edit the title and comment on the editing page for the cellular phone (Figure 6, Figure 7).

The positional data are taken out from the photograph which was sent from the cellular phone using the Exif tags. Though the Exif tags are often used to sort out the photographs [5] [6], it is used in this system to construct the virtual world using the positional data. Therefore, when the photographs that were taken by digital camera were sent to the server with the positional data using the Exif tags, they can also be located in the virtual world automatically. Although the KEITAI-Space [7] constructs a virtual space in the cellular phone, this system generates the virtual world that can be experienced in the immersive projection environment.

\section{Immersive Virtual World}

In the immersive projection display, the user can experience the three-dimensional virtual world with high quality of presence [8] [9].

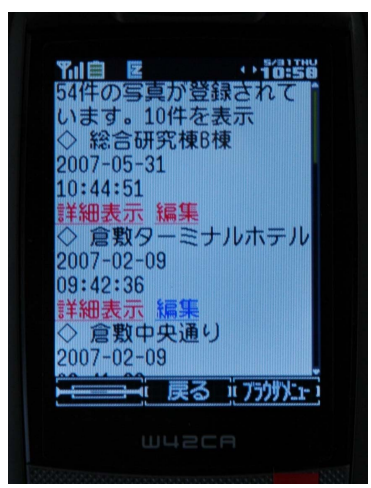

Figure 6. BBS in cellular phone

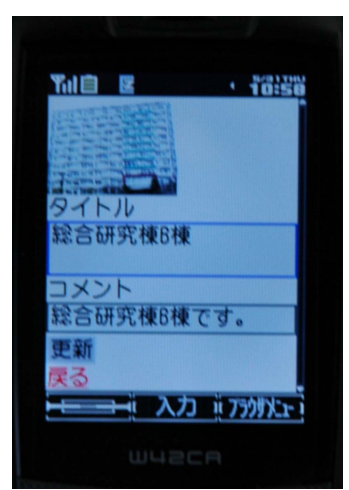

Figure 7. Editing form in cellular phone

\subsection{CS Gallery}

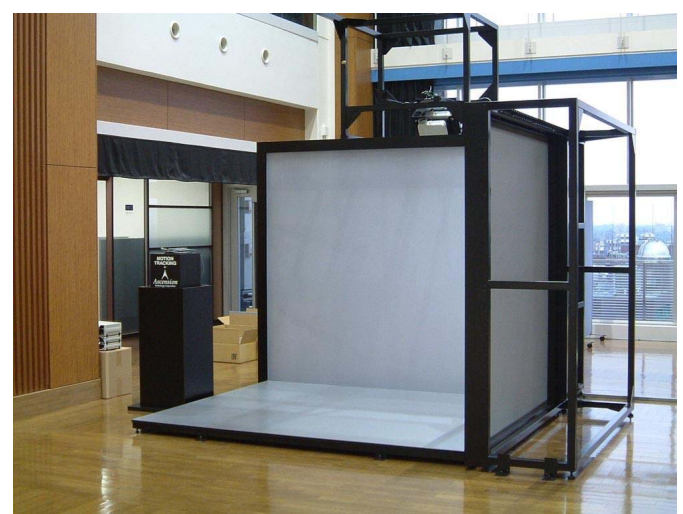

Figure 8. CS Gallery

In order to visualize the virtual world, a CAVE type immersive projection display named CS Gallery was used [10]. Figure 8 shows the CS Gallery. The CS Gallery is composed of three screens, placed at the front, on the right side, and floor side. This system displays passive stereo images by using the circular polarized filters. In this system, three PCs are used to render the image and one database server is used to store the photographs and map data. The database server is also used as the web server, and the map BBS function runs on the server computer. Apache is used for the web server function, and MySQL is used for the database server function.

Each of rendering PC corresponds to the front, right, and floor screens of the CS Gallery. Each PC sends SQL statement to the database server using the ODBC protocol, and renders the image contents using the received data. In this case, NFS is used to read the image data stored in the database server.

In addition, the positional data of the user's viewpoint is measured by one rendering PC, and it is sent to the other rendering PCs with the command data 
inputted by the keyboard. By sharing the information, the synchronization and the consistency of the displayed images can be realized among three screens, and the user can see the images without discontinuity at the corner parts of the screen.

\subsection{Construction of Virtual World}

As an initial state, the empty world in which only a texture image of the map was placed on the floor is prepared. As for the texture image of the map, images of SGI format are used. These map images are also stored in the database, and the virtual world is displayed by specifying the user name, the area of map, and the category. Then, the photograph images are placed on the map according to the latitude and longitude by transforming them into the virtual world's coordinates.

The parameter data that is used to display map, such as center position in the virtual world and reduced scale, are also stored in the database. The virtual world is displayed in the reduced scale, because it is hard for the user to walk through the vast virtual world when it is displayed in full scale. And the position where the photograph is placed on the map is calculated from these parameter data.

Since the place names printed on the map are unreadable, the character strings of the place names are also stored in the database and they are displayed in the space perpendicular to the floor as shown in Figure 9.

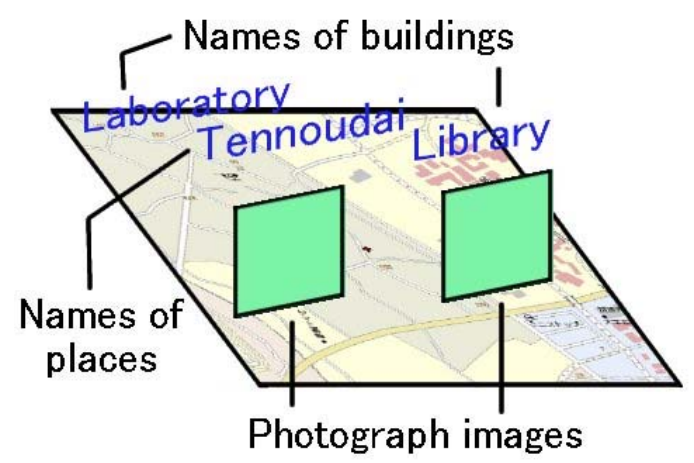

Figure 9. Construction of virtual world

\subsection{Operation in Virtual World}

The example of the constructed virtual world is shown in Figure 10. The user in the virtual world uses a small keyboard (ELECOM TK-U77MP) as the interface device and can move freely in it by the walkthrough operation. The nearest photograph to the user is visualized being enlarged automatically, and the comment about the enlarged photograph is displayed when the user selects the comment window using the key command as shown in Figure 11. In this window, the user can also write a comment to the photograph. Since the comment written in the virtual world can be seen by other users, this function can be used as the VR BBS. In addition, since the inputted comment is uploaded to the database, it is also reflected to the map BBS function used on the web.

In the virtual world, when the user selects the photographs, they are stored in the bookmark list and they can also be accessed from the PC. When the listed photographs are displayed in the virtual world, the numbers of order in the list are shown above the photograph images (Figure 12). The user can change the order of the listed photographs or delete a photograph from the list. In this system, the user can make multiple lists for each area of the displayed map. Since the user can browse listed photographs on a cellular phone and a PC as well as in the virtual world, he can refer to the listed data selected in the virtual world after going to the outdoor field.

In the VR BBS, it is possible for the user to write his opinion or thoughts about the photographs placed on the map while experiencing them in the virtual world. Since the other users can also use the BBS function in the virtual world, they can communicate with each other while sharing the information in the virtual world. Therefore, this system can be used as an experience-type BBS compared to the ordinary BBS used on the PC or the cellular phone. In this system, since the virtual world constructed by other users contains a lot of valuable data for a user, he can obtain necessary information about the place before visiting there. In addition, the more users use this system, the more information is stored in the virtual world. Therefore, the system itself can be extended automatically.

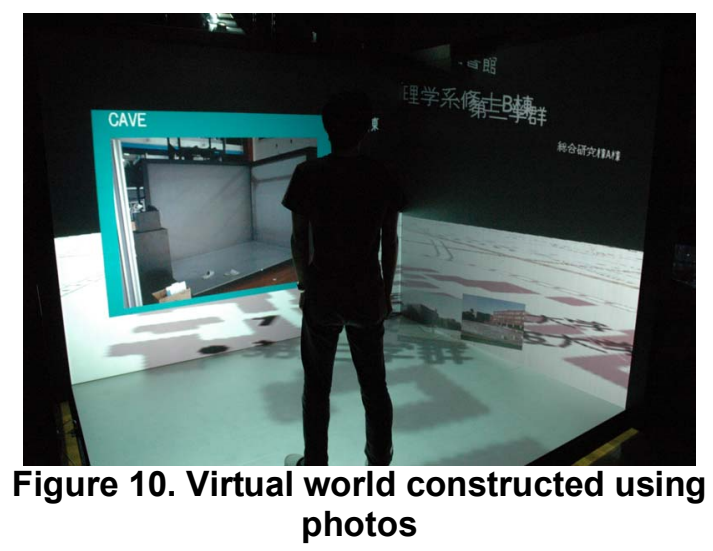




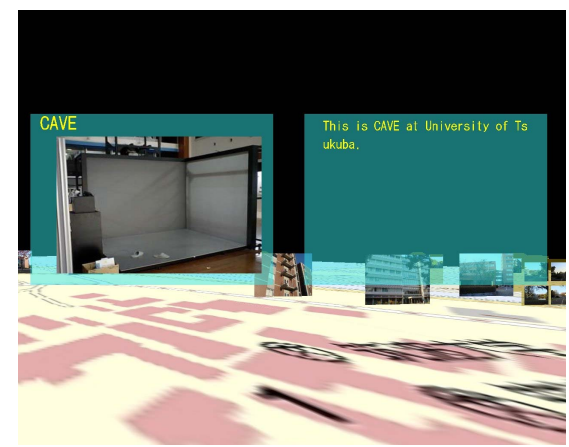

Figure 11. Writing comment using VR BBS

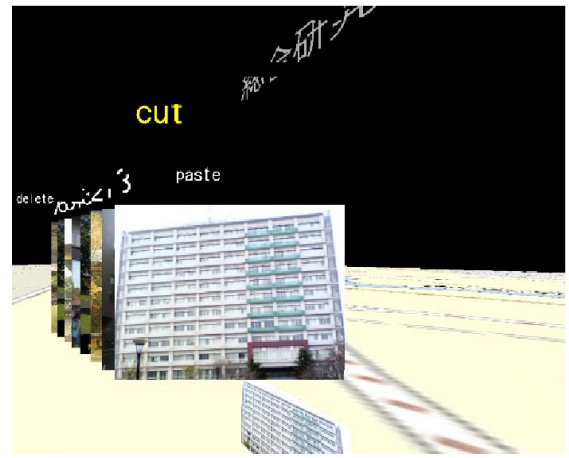

Figure 12. Listed photograph

\subsection{Examples of Application}

In this system, the user who is traveling in the real world can post the photograph with the positional data and comment by using the cellular phone, and the virtual world can be constructed and updated automatically. And the user in the virtual world can not only communicate with the traveler offline but also understand the traveler's experience through the photographs and the comments. The user who is traveling can also browse the comments using the cellular phone and send a reply to the comments. Therefore, the user can communicate with the remote user while sharing the experience between the real world and the virtual world.

In addition, the user can relive the experience of the travel in the virtual world that was constructed using the photographs sent from the cellular phone after coming home from the travel.

\section{Experiments}

In this system, information such as the photograph and the comment is expressed in the virtual world that is constructed using a map. In such a system, it is important that the user can recognize the positional information. In this study, we conducted an experiment to evaluate the user's spatial recognition.
(1) Experimental method

In this experiment, we investigated learning levels of the subjects when they learned the spatial information of the old buildings in Kyoto by using two kinds of methods. In one case, the subjects read a guidebook to learn about the old buildings, and in the other case, they used VR BBS while experiencing the virtual world.

In the case of the guidebook, we selected the items of 25 buildings in the guidebook and put marks on them. In the guidebook, a photograph and an explanation for each building are printed on the different pages from the maps. The subjects were asked to memorize the photograph, the explanation and the location on the map of each building. In the case of using the VR BBS, the virtual world was constructed by uploading the information of 25 buildings that were different from the items used in the guidebook. The name and the explanation reprinted from the guidebook were displayed with the photograph of each building. The subjects were also asked to memorize them with the location while walking-through the virtual world.

The number of subjects was six. The subjects were given 20 minutes of learning time in both cases and they were asked to answer the question after learning. In this question, the subjects were asked to connect the correct relation among the photograph, explanation and location on the map.

(2) Results

Figure 13 and Figure 14 show the results. The answers of the subjects included "correct", "incorrect", and "partial correct". The partial correct answers was further classified into three groups, "photograph and location are correct", "explanation and location are correct", and "photograph and explanation are correct". Since a lot of numbers of old buildings were given, some of the subjects were not able to answer all the questions. In this experiment, we evaluated the partial correct answer of "photograph and location are correct" and "explanation and location are correct" to investigate the user's learning level about the spatial information. In addition, the wrong answer about the location in which the distance error was less than $1 \mathrm{~km}$ was evaluated "roughly correct" in Figure 14. In these graphs, the average and the standard deviation of the numbers of correct answer and incorrect answer when the guidebook and the VR BBS were used are shown. Horizontal axis indicates the types of results, and vertical axis is the number of answers for each result.

(3) Discussions

From the results of this experiment, the number of correct answers when the VR BBS was used was more than the number of correct answers when the guidebook was used. T-test was applied to analyze the 
result statistically. Although there was no significant difference between the guidebook and the VR BBS about perfectly "correct" answers, the significant difference was found at 5\% about the answer including "partial correct" and at 1\% about the answer including "roughly correct".

Therefore, we can understand that this system can effectively be used to acquire knowledge recognizing spatial relationship. Some subjects reported that it was easy for them to understand the position of each building because they can overlook the map in the virtual world. Namely, they could learn the positional information connected to the location on the map by walking through the virtual world in the immersive projection environment.

On the other hand, some subjects said that they felt sick when they memorized the information about the old buildings in the virtual world. In this experiment, since the map of wide area of Kyoto was displayed, the user must walk-though the long distance in the virtual world. In addition, it can be thought that the experimental task in which the subjects must read characters and sentences in the virtual world for a long time have greatly influenced. This problem must be solved in the practical applications.

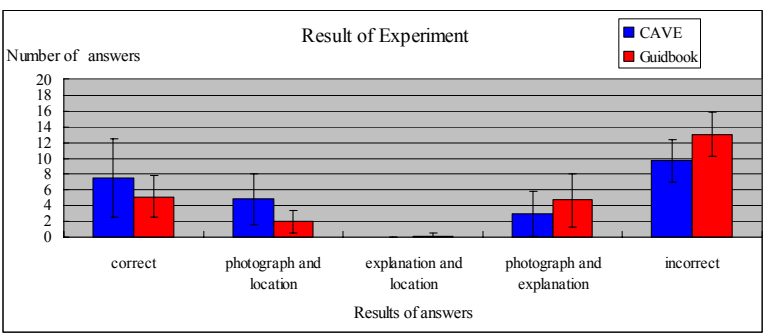

Figure 13. Result of experiment

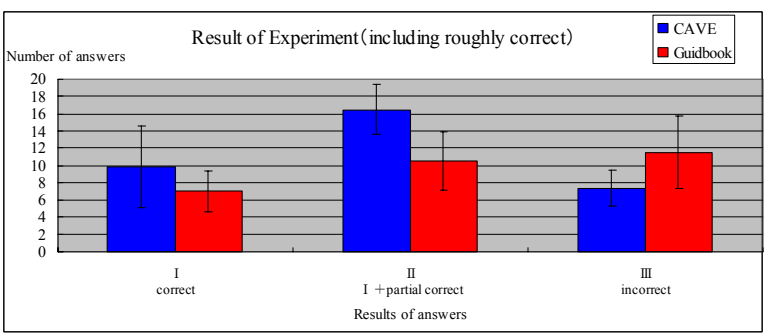

Figure 14. Result of experiment

\section{Conclusions}

In this study, VR BBS in which the users can share information and exchange thoughts using the map BBS function on the $\mathrm{PC}$, cellular phone and in the immersive virtual world was developed. In this system, the virtual world is constructed based on the positional data of the uploaded photographs. And the user can read and write comments for the photograph images in the immersive virtual world.

In the current system, only the map and the photograph data are used to construct the virtual world. In future works, we are planning to construct high presence virtual worlds by using three-dimensional objects as well as to expand the BBS function.

In addition, we will solve the problem of the VR sickness so that the user can use it for a long time, by adjusting the size of the displayed characters or controlling the speed of the walk-through function.

\section{References}

[1] YouTube: http://www.youtube.com/

[2] Flickr: http://www.flickr.com/

[3] Google Maps: http://maps.google.com/

[4] KASHIMIR 3D: http://www.kashimir3d.com/

[5] Lei Zhang, Le Chen, Feng Jing, Kefeng Deng, and Wei-Ying Ma, "EnjoyPhoto-A Vertical Image Search Engine for Enjoying High-Quality Photos", Proceedings of the $14^{\text {th }}$ annual ACM International conferences on Multimedia MULTIMEDIA '06, 2006, pp. 367-376.

[6] Noah Snavely, Steven M. Seitz, and Richard Szeliski, "Photo tourism: exploring photo collections in 3D", ACM SIGGRAPH 2006 Papers SIGGRAPH '06, 2006, pp. 835-846.

[7] Taro Nakao, Masahiko Tsukamoto, and Takefumi Ogawa, Shojiro Nishio, "KEITAI-Space: Photo-Based Shared Virtual Space on Cellular Phones", Proceedings of the $18^{\text {th }}$ International Conference on Advanced Information Networking and Application, 2004, pp.358-363.

[8] Michitaka Hirose, Tetsuro Ogi, Shohei Ishiwata, and Toshio Yamada, "Development and Evaluation of Immersive Multiscreen Display 'CABIN", Systems and Computers in Japan, Scripta Technica, Vol.30, No.1, 1999, pp.13-22.

[9] Carolina Cruz-Neira, Daniel J. Sandin, and Thomas A. DeFanti, "Surround-Screen Projection-Based Virtual Reality: The Design and Implementation of the CAVE", Proceedings of SIGGRAPH93, 1993, pp. 135142.

[10] Tetsuro Ogi, Takaya Uchino, "Dynamic LoadBalanced Rendering for a CAVE System", Proceedings of the ACM symposium on Virtual reality software and technology VRST '06, 2006, pp.189-192. 